\title{
Reseña: Brown, W. (2016) El pueblo sin atributos: La Secreta Revolución del Neoliberalismo. Madrid: Malpaso ediciones.
}

El pueblo sin atributos: La Secreta Revolución del Neoliberalismo es, sin lugar a dudas, una de las mayores contribuciones que se han hecho al campo de la teoría política en los últimos años por su rigurosidad conceptual, originalidad intelectual y meticuloso análisis de la realidad. Wendy Brown publicó en 2015 la versión inglesa bajo el título Undoing the Demos: Neoliberalism's Stealth Revolution. La traducción al castellano llegó un poco más de un año después. Lamentablemente, no he podido hacerme con una copia de la versión traducida, pero innegablemente este libro merece una revisión para alentar al lector a pasearse por sus valiosas páginas.

Este texto encaja sin ningún problema en esa categoría de libros que realizan un sobrio análisis del presente y se convierten instantáneamente en una lectura obligatoria para cualquier persona interesada en entender el panorama político al que nos enfrentamos o simplemente para cualquiera interesado en el desarrollo de la teoría política. El objetivo principal del libro es explicar la forma en que el neoliberalismo deshace y rehace cualquier principio o valor democrático que subyace al Estado, la sociedad y la producción de subjetividades al tomar como único principio operativo la reproducción de los principios de mercado en todas las esferas vitales. De esta forma, el avance en la utopía neoliberal implica un destino oscuro para la democracia una vez que el valor de la vida humana es reducido meramente a su aspecto económico. Por tal motivo, para Brown, 'el neoliberalismo es la

\footnotetext{
* Sebastián Raza (Licenciado en Sociología, Pontificia Universidad Católica del Ecuador) se desempeñó como ayudante de cátedra bajo la supervisión de Emilio Cerezo desde 2012 hasta 2016. Actualmente cursa estudios de Maestría en Sociología en la Universidad de Warwick, Reino Unido.

$\bigotimes$ :s.raza-mejia@warwick.ac.uk
} 
racionalidad por medio de la cual el capitalismo finalmente logra tragarse a la humanidad' (44).

\section{Argumento y Estructura}

El libro se divide en dos partes, compuestas de tres capítulos cada una. En la primera se exponen los principios teóricos con los cuales se debe entender al neoliberalismo; mientras, en la segunda, se analizan los cambios instaurados por la racionalidad neoliberal en el derecho, la educación y los principios de ciudadanía, en el contexto estadounidense. Con base en $E l$ Nacimiento de la Biopolítica de Michel Foucault, Brown considera que el principio teórico rector para comprender el neoliberalismo es verlo como una 'racionalidad política' que, en lugar de oponer el Estado al Mercado, utiliza al primero como un mecanismo para la economización de todas las esferas de lo social y subsumirlas bajo la lógica de la competencia y el cálculo económico. Como resultado de esta operación, el ser humano queda reducido a la figura del homo economicus que constantemente debe maximizar sus rendimientos en interacciones de mercado. En palabras de Brown (31), 'la racionalidad neoliberal disemina el modelo del mercado a todos los dominios y actividades - aun cuando no haya dinero involucrado - y concibe al ser humano exclusivamente como actor económico...como homo economicus'. La consecuencia de esta doble operación es que los principios democráticos que fundamentan varias prácticas humanas son socavados en favor valores y prácticas económicas.

En el primer capítulo, Brown se encarga de explicar en qué consiste específicamente la economización neoliberal del ser humano y las esferas sociales. En contraste con el liberalismo clásico, el homo economicus no se concibe como un ser de intercambio e intereses, sino de competencia e inversiones en sí mismo en tanto capital humano que está orientado a la 
optimización personal. En este sentido, argumenta Brown, los agentes se comportan como emprendedores de sí mismos en busca de financiamiento para mejorar su rentabilidad en cada interacción y toma acciones de ocio, consumo, educación, etc., solo en vistas a la posibilidad de que estos aumenten sus réditos futuros. En palabras de Brown, 'el reinado normativo del homo economicus en todas las esferas significa que no hay otras motivaciones, impulsos o aspiraciones aparte de las económicas' (44). De esta forma, el mercado y la competencia se vuelven un eje normativo según el cual las acciones institucionales e individuales se configuran y juzgan. Consecuentemente, al entender al neoliberalismo como una forma de racionalidad política, no se puede afirmar que este dicta un conjunto preciso de políticas económicas sino una forma particular de modelar el Estado, la sociedad y los sujetos a partir de la economización de la vida social: "la economía es al mismo tiempo modelo, objeto y proyecto’.

En el segundo capítulo, Brown retoma y critica el análisis foucaultiano sobre el neoliberalismo. Ambos coinciden en que el neoliberalismo debe ser entendido como una racionalidad política que sitúa al mercado como el sitio último de 'verdad' y legitimación de cualquier forma de gobierno y acción gubernamental que economiza todo lo que toca y obliga a los individuos a convertirse en homo economicus en la totalidad de sus vidas. En otras palabras, la racionalidad política neoliberal hace del Estado un regulador del mundo social a partir de la lógica del mercado al diseminar principios de competencia. Para los neoliberales, tanto en la versión de Hayek como en la versión Ordoliberal, la competencia es esencial pero no natural; por tanto, debe ser fomentada y corregida desde el exterior al mantener, reforzar y reproducir sus principios operativos. La inversión del liberalismo clásico es sutil pero clara: el mercado deja de ser el límite exterior de cualquier forma de gobierno y se transforma en el principio interior de toda acción gubernamental. La economía no se 'libera', sino que se observa y propicia 
desde acciones gubernamentales que fomentan prácticas económicas de competencia.

Otro cambio 'tectónico' en relación al liberalismo clásico es que el fundamento del mercado ya no es el intercambio entre iguales, sino la competencia entre desiguales: un intercambio basado en la igualdad presupone como resultado de la operación "satisfacción", basada en la equidad de los productos intercambiados, mientras que la competencia siempre tiene como prerrequisito y resultado la desigualdad de los participantes. De esta forma, argumenta Brown, la desigualdad se torna en un principio normativo para la economización de todas las esferas de la vida social. Como resultado de este cambio, se introduce un principio que socava prácticas de solidaridad y justicia que subyace en las instituciones democráticas: en toda relación social hay 'ganadores' y 'perdedores', y para que la competencia funcione debemos aceptar que una proporción de individuos debe perder. Así, 'la racionalidad política neoliberal no solo economiza en el sentido de monetizar toda conducta o relación social, sino que, de manera más radical, las formula en un marco exclusivamente económico que tiene dimensiones ontológicas y epistemológicas' (62). El neoliberalismo no opera como intercambio entre dos iguales sino en términos de competencia entre dos capitales (humanos). Esta configuración permite re-imaginar todas las esferas sociales en términos de competencia y cálculo, aun cuando no haya un mecanismo monetario.

Al final del segundo capítulo, Brown inicia las críticas a Foucault y empieza a delinear su comprensión de democracia. La crítica de Brown a Foucault se basa en que, si bien El nacimiento de la biopolítica nos permite comprender las características del neoliberalismo y del homo economicus, no ofrece pistas para comprender los cambios introducidos en el ámbito social, a menos que se consideren las figuras humanas que entran 'en peligro de extinción'. En este sentido, Brown detecta que un problema mayor en la obra 
de Foucault es la omisión de un demos (pueblo), fundamentado en la imagen de homo politicus que reclama no ser gobernado y lucha por gobernarse a sí mismo. Si el homo economicus es el sujeto de la racionalidad neoliberal, la única forma de materializar prácticas democráticas es mediante la existencia de un homo politicus en tanto 'sujeto que se gobierna a sí mismo a través de autonomía moral y gobierna con otros mediante la soberanía popular' (79). De esta forma, cuando el sujeto económico reina en lo social, lo que desaparece es la forma de subjetividad y las condiciones de posibilidad que pone en marcha prácticas democráticas.

En el tercer capítulo, con el fin de demostrar que la reducción del humano a puro agente económico es una característica exclusiva del neoliberalismo, Brown (87-99) realiza un análisis genealógico en el que demuestra que, en todo pensamiento político, desde Aristóteles hasta Mill, pasando por Locke, Hegel, Smith y Bentham, ha presupuesto la existencia de un homo politicus y ha mostrado sus reservas frente a los perversos efectos morales del homo economicus. En otras palabras, Brown demuestra que el pensamiento liberal y político presupone de una u otra manera un sujeto capaz de juicio valorativo que se gobierna a sí mismo y busca no ser gobernado, que actúa en razón del interés comunitario y del reconocimiento mutuo, que pasa por procesos de deliberación y soberanía. Así, Brown concluye que 'el homo economicus asciende y expande su dominio en la modernidad Euro-Atlántica, pero el homo politicus se mantiene vivo e importante a través del tiempo - lleno de demandas y expectativas, como el lugar de la soberanía política, libertad y legitimidad' (98).

Sin embargo, el neoliberalismo no está dispuesto a hacer las concesiones que sus antepasados liberales hicieron al homo politicus. Con la final derrota de este último, Brown (78) argumenta que 'la autonomía moral $\mathrm{y}$, por tanto, la base para la soberanía individual desaparece; así como el espacio y significado de la ciudadanía política se contrae'. Mientras que 
el liberalismo siempre mantuvo 'el lenguaje y la promesa de equidad política, inclusión, libertad y soberanía popular' (44), la proliferación de los principios neoliberales se delinea como una barrera para la ejecución de acciones políticas autónomas, dado que el neoliberalismo es ciego frente a otras formas de ser-en-el-mundo basadas en principios no económicos ni de competencia o de cálculo racional basado en el egoísmo exclusivista.

Los capítulos 4, 5 y 6, que componen la segunda parte del libro, se encargan de exponer los cambios introducidos por la diseminación de la racionalidad neoliberal en esferas vitales que hasta ese momento no eran económicas por medio de la racionalidad neoliberal. El capítulo 4 muestra que las prácticas de gobernanza, una vez alineadas con los preceptos neoliberales, adquieren un matiz propio en términos de disciplinamiento de sujetos y manejo del Estado. La gobernanza neoliberal no se preocupa del consenso político sino de lo que el mercado demanda. De esta forma, el consenso neoliberal es producido no a partir de un demos que busca gobernarse a sí mismo, sino por la obediencia a las leyes del mercado en tanto se considera al ser humano exhaustivamente como homo economicus. Así, la gobernanza neoliberal mezcla los léxicos políticos y empresariales e introduce prácticas económicas en el Estado como el 'benchmarking', el sacrificio individual e incluso grupal en aras de la salud económica a través de políticas de austeridad, el salvataje de empresas que suponen un bien para la economía en general, etc. La conducción del Estado deja de tener un contenido democrático y sus acciones se califican a partir de índices de Mercado, inversiones y competitividad, no a partir de principios de equidad, libertad e inclusión. En este sentido, Brown afirma que 'la conducción del gobierno y la conducción de la empresa son ahora fundamentalmente idénticos' (27). 
En el capítulo 5, Brown se encarga de demostrar cómo la corte suprema estadounidense se ha saturado de lenguaje económico y ha dejado de lado valores inherentes al homo politicus. Al gobernar para reproducir e introducir el principio de competencia, la ley se convierte en un elemento táctico del mercado y no en una expresión de los derechos de los individuos. La ley, en esta versión, no se utiliza para alcanzar derechos sociales o planificación para mejorar la vida de la población, sino como médium para facilitar el juego económico entre empresas o individuos. El capítulo 6, por su parte, demuestra cómo el neoliberalismo atenta contra la criticidad que supone la educación superior, y destruye así la base de ciudadanía ilustrada que necesita cualquier práctica democrática.

El punto central de estos tres capítulos es que la gobernanza neoliberal se formula en un lenguaje anti-político sin referencia a la democracia y se convierte en la lingua franca del Estado, las universidades y virtualmente toda institución social. Brown demuestra que es un error pensar que, a través de prácticas neoliberales, podemos expandir la democracia. Este libro es urgente en el panorama actual latinoamericano en el que los partidos de derecha, con propuestas neoliberales, buscan presentarse como la solución democrática ante los autoritarismos de los gobiernos de turno. Brown demuestra que es urgente reconocer que, en el neoliberalismo, 'los Estados están subordinados al mercado, gobernados por el mercado, y ganan o pierden legitimidad a partir de las vicisitudes del mercado' (108). El manejo de la ley, el Estado, los derechos y la educación dejan de tener como criterio la justicia, la protección del ciudadano o el balance de intereses y su único objetivo es el crecimiento económico, la calificación del crédito y el posicionamiento competitivo. De esta forma, el ciudadano se vuelve inminentemente gobernable y sacrificable desde el punto de vista del Estado. En el epílogo, Brown regresa sobre este punto y demuestra cómo el sacrificio individual demandado por el mercado y ejecutado por el mercado 
(a través de políticas de austeridad) nos recuerdan la inseguridad, desdemocratización y fragilidad inherente al neoliberalismo.

El libro de Brown no hace una apología de la democracia liberal, pero reconoce que su vocabulario y subjetividades pueden ser la base para el desarrollo de una crítica contra el capital y pueden gestar prácticas democráticas más radicales. Lejos de defender ciegamente la democracia liberal, lo que preocupa a Brown es la economización de la sociedad, que destruye el terreno apto para que cualquier política radical tenga lugar pues se pierde el principio de un pueblo que busca autogobernarse. En sus palabras, 'la democracia no es ni la panacea ni la forma completa de la vida política. Sin embargo, sin ella perdemos el lenguaje y el marco por el cual somos los dueños del presente y podemos hacer nuestro futuro, el lenguaje y el marco con el cual hacemos frente a otras fuerzas que quieren manejar nuestro futuro' (210).

\section{Limitaciones y nuevas líneas argumentativas}

Una de las mayores limitaciones del libro de Brown es que analiza exclusivamente el contexto estadounidense. A pesar de que Brown reconoce que "en sus varios encuentros con distintas culturas y tradiciones políticas, el neoliberalismo toma diversas formas y genera contenidos, detalles normativos distintos y diferentes léxicos' (48), poco o nada se dice de las transformaciones introducidas en distintos lugares del mundo por la racionalidad neoliberal. Este punto es significativo, al menos en dos aspectos. En primer lugar, en tanto fenómeno global, el neoliberalismo implica diferentes formas de inserción con el capital global y, por tanto, distintas demandas del mercado. Esta diferenciación se traduce en distintas reconfiguraciones de las esferas educativas, del Estado y de la ley en distintos 
lugares del planeta. En otras palabras, la reconfiguración neoliberal de la sociedad, el Estado y las subjetividades está condicionada por la forma en que los países se insertan en el capitalismo global y, por tanto, no es posible generalizar inmediatamente todos los hallazgos de los capítulos 4,5 y 6 , aunque en general ofrezcan una clara tendencia. En este sentido, se podrían realizar análisis del modelo educativo basado en el 'voucher' que ha sido invocado por candidatos de derecha, así como la idea de zonas francas en la medida en que son formas de gobernanza neoliberal específicas de cierto modo de inserción en los mercados internacionales.

En segundo lugar, el tono apocalíptico del libro de Brown también puede ser un producto de esta limitación geográfica, si tomamos en cuenta la resistencia contra las políticas neoliberales en América Latina que empiezan en los años 90 y que, a pesar de haberse atenuado a lo largo de los años, han logrado cristalizarse en algunas prácticas gubernamentales. Como ejemplo se puede citar la denuncia de inconstitucionalidad de 12 tratados bilaterales de protección recíproca de inversiones realizada por la Asamblea ecuatoriana en el 2017 porque apela a que los juzgados internacionales se enmarcan en los intereses del capital y no de los países involucrados. Este es un ejemplo de resistencia a formas de gobernanza neoliberal global que se preocupan exclusivamente por atraer inversiones y el posicionamiento competitivo mediante la subordinación a los mercados. Tal denuncia demuestra que en específicos puntos geográficos hay luchas contra la desdemocratización neoliberal y levanta la pregunta de hasta qué punto se debe pensar la existencia de una racionalidad política pos-neoliberal que emerge a partir de luchas re-democratizadoras frente a la racionalidad neoliberal. Estas dos líneas argumentativas deberán, sin duda alguna, tomar como referencia el libro de Wendy Brown pero rebasan sus alcances. 